\title{
The Kidney-Heart Connection in Obesity
}

\author{
Almudena García-Carrasco Adriana Izquierdo-Lahuerta Gema Medina-Gómez \\ Área de Bioquímica y Biología Molecular, Departamento de Ciencias Básicas de la Salud, Facultad de Ciencias de la \\ Salud, Universidad Rey Juan Carlos, Madrid, Spain
}

\author{
Keywords \\ Obesity · Cardiorenal metabolic syndrome · Kidney · Heart · \\ Endothelium hemodynamic changes
}

\begin{abstract}
There is a strong relationship between the kidney and the heart, where if one of these organs fails, so does the other, in the so-called cardiorenal syndrome (CRS). Besides, there are also interactions with the rest of the body leading to a metabolic state that establishes a feedback loop that is perpetuated. The CRS is characterized by hemodynamic changes, activation of neuro-humoral systems, natriuretic peptides, and changes in mineral metabolism. In this scenario, the kidney and heart, connected by a dysfunctional endothelium, inevitably fail. In obesity, this syndrome is exacerbated due to the complications of adipose tissue dysfunction, in the socalled cardiorenal metabolic syndrome (CRMetS). Obesity promotes adipose tissue dysfunction because it exceeds lipid storage capacity and leads to a lipotoxic state, characterized by inflammation, hypertension, insulin resistance and dyslipidemia, oxidative stress, and hyperuricemia, among others, that affect different organs other than the adipose tissue. In addition, the pro-inflammatory gut microbiota present in obese patients releases uremic toxins, contributing to oxidative stress and inflammation, perpetuating and accelerating the progression of this pathology. In this article, we describe the contribution of obesity, the factors and mechanisms implicated in the development of the CRMetS.
\end{abstract}

Despite the great knowledge about the CRS, more research is needed to characterize the CRMetS given the global obesity epidemic.

(c) 2021 S. Karger AG, Basel

\section{Introduction}

The relation between the heart and kidney was first described by Robert Bright in 1836, but until 2005, the term cardiorenal syndrome (CRS) was not properly defined as "disorders of the heart and kidney whereby acute or chronic dysfunction in one organ may induce acute or chronic dysfunction of the other" (Table 1). There are risk factors that co-exist in both the kidney and heart, which could explain this pathology. Moreover, recent work has highlighted the endothelium dysfunction as a possible link leading to the failure of the two organs. Indeed, maintaining a functional endothelium capable of responding adequately to hemodynamic changes is key to prevention of CRS [1].

In this context, since the emerging pandemic of obesity, the effects of overweight/obesity on the heart and kidney have also been studied. The presence of common features with metabolic syndrome, besides others like mi-

Contribution from the CME course of the DIABESITY Working Group of the ERA-EDTA, Alcorcón (Madrid), Spain, November 15-16, 2019.

karger@karger.com www.karger.com/nef

(c) 2021 S. Karger AG, Basel

Karger'
Correspondence to:

Adriana Izquierdo-Lahuerta, adriana.zquierdo@urjc.es

Gema Medina-Gómez, gema.medina@ urjc.es 


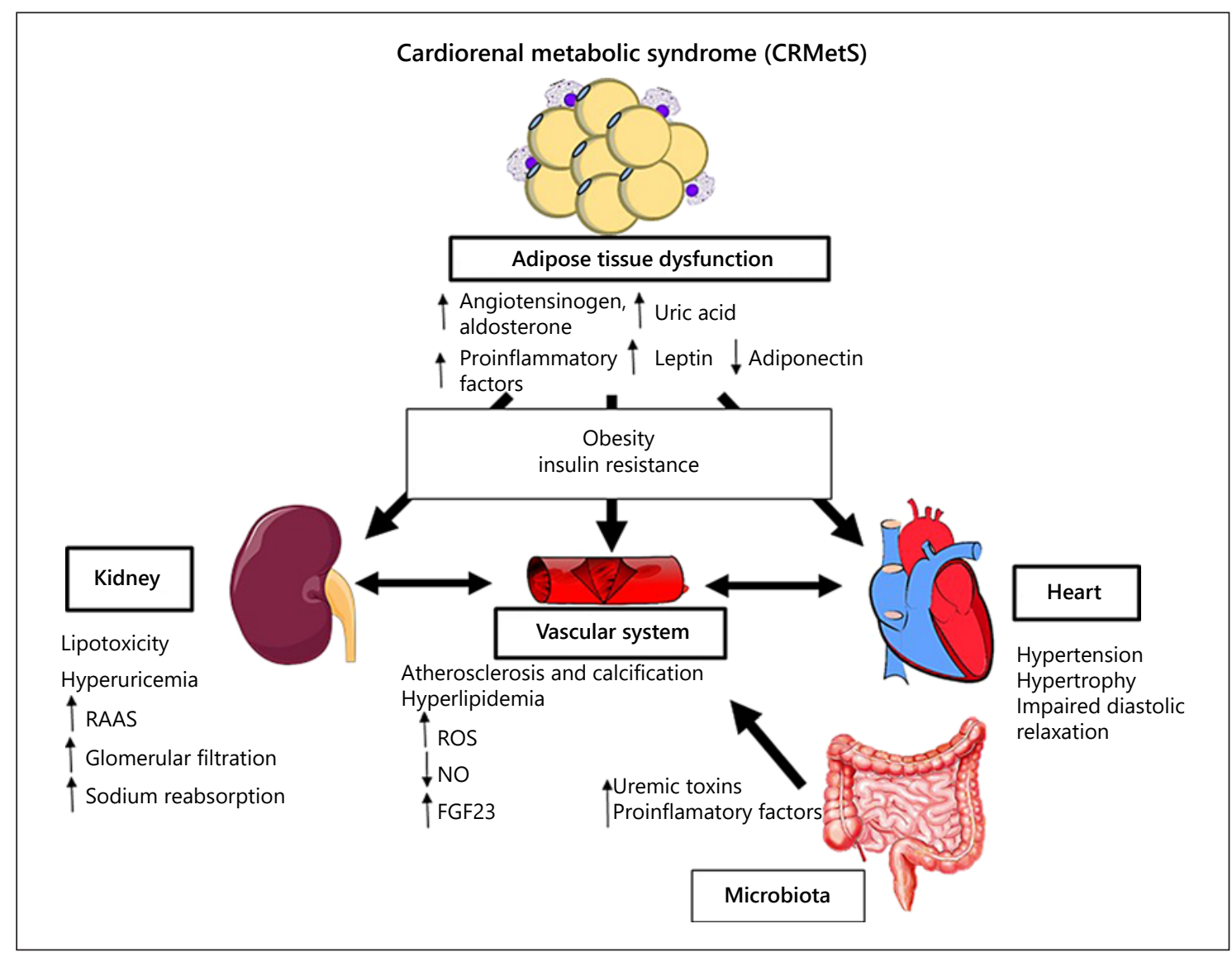

Fig. 1. Implications of different tissues in CRMetS. CRMetS, cardiorenal metabolic syndrome; FGF23, fibrosis growth factor 23; NO, nitric oxide; RAAS, renin-angiotensin-aldosterone system; ROS, reactive oxygen species.

croalbuminuria, in a CRS background has been defined as cardiorenal metabolic syndrome(CRMetS) [2] (Fig. 1). In this review, we look at the factors and mechanisms that cause obesity to lead to worsening and accelerating damage, and we reinforce to consider this as a new type of CRS, the CRMetS (Table 1).

\section{Mechanisms and Factors Involved in CRMetS}

\section{Adipose Tissue Dysfunction in Obesity}

In obese patients, as the white adipose tissue (WAT) expands, a recruitment of inflammatory cells occurs and promotes changes in the vasculature and the extracellular matrix to allow oxygenation and mobilization of nutrients. If obesity and inflammation are sustained, these adaptive homeostatic mechanisms fail, leading to WAT dysfunction characterized by impaired secretion of adipokines, abnormal lipid storage and adipogenesis, exac- erbated matrix deposition, and insulin resistance (IR) [3] (Fig. 1). In this context, the lipid storage capacity of WAT is exceeded, and the excess lipid is released into the blood and is captured by different organs, including the kidney and heart, that begin to develop IR and dysfunction, as result of the lipotoxic process.

\section{Kidney and Heart Failure, and Endothelial Dysfunction in CRMetS}

During obesity, the individual has to reach larger metabolic demands. This situation is translated into several changes: 1) an increase in the blood volume and heart size; 2) relaxation abnormalities before a contractile dysfunction, and 3) the presence of IR, which leads to an impairment in diastolic relaxation through oxidative stress, inflammation, and decreased levels of nitric oxide (NO). Also in the kidney, where a characteristic glomerular hyperfiltration is established due to an increase in the intraglomerular pressure, activation of the renin-angiotensin- 
Table 1. "Classic" CRS classification, depending on the first affected organ, into 5 groups, and new type of CRS

\begin{tabular}{llll}
\hline Type & Name & Primary organ affected & Secondary organ affected \\
\hline 1 & Acute CRS & Heart failure & Acute kidney injury \\
\hline 3 & Chronic CRS & Chronic heart failure & CKD \\
\hline 4 & Acute renocardiac syndrome & Acute kidney injury & Acute heart failure \\
\hline 5 & $\begin{array}{l}\text { Chronic renocardiac } \\
\text { Syndrome }\end{array}$ & CKD & Chronic heart failure \\
\hline & Secondary CRS & Systemic & Heart failure/kidney injury \\
\hline
\end{tabular}

aldosterone system (RAAS) by the adipose tissue and sympathetic nervous system activation to maintain plasma flow. This RAAS activation generates a large quantity of reactive oxygen species and increases the sodium reabsorption, perpetuating the damage $[3,4]$. Recently, it has been described that the factors of higher cardiometabolic risk, such as hypertension, hs-C-reactive protein, systolic blood pressure, waist circumference, body fat percentage, and visceral area, appear precisely in individuals with the highest body mass index (BMI) [4].

Diet is another important point. Along with the number of calories on a diet, the composition is also relevant. In this regard, the high consumption of fructose leads to the synthesis of higher levels of uric acid by the adipose tissue. It has been shown that hyperuricemia impairs kidney function, which will result in renal failure and the inability to filter the uric acid, closing the cycle. Besides, vascular stiffness, a RAAS activation, an increase in the blood pressure, inflammation, and hyperinsulinemia are also observed [5]. Hyperinsulinemia can reduce renal excretion of uric acid as insulin can stimulate the urate-anion exchanger and/or the sodium-dependent anion cotransporter in the brush border membranes of the renal proximal tubule and increase urate reabsorption [6].All these ingredients make a great recipe for characteristic hypertension during obesity, which results in left ventricular hypertrophy and impaired cardiac diastolic relaxation. Beyond this impact, the systemic activation of the RAAS inhibits the endothelial production of $\mathrm{NO}$ and its bioavailability in cardiovascular tissues [7]. Furthermore, the inhibition of the endothelial NO synthase contributes to IR, hypertension, and defects in mitochondria and fatty acid metabolism [8].
Mitochondrial alterations are associated with obesity, like oxidative stress or alterations in mitochondrial biogenesis, decrease mitochondrial oxidative capacity, contributing to CRMetS by increasing IR, cholesterolemia, or hypertension [8]. One of the main players in lipid handling in an obese context is the peroxisome proliferatoractivated receptor $\gamma$ and its co-activator, known as PGC$1 \alpha$, responsible for the mitochondria biogenesis and function. Defects in heart muscle contractility have been shown in a model of PGC-1 $\alpha$ null mice [8]. Not just the functionality but also the mitochondrial number affects lipid handling, leading to their accumulation in the cell and contributing to the IR. Angiotensin II also contributes to the oxidative stress by increasing the expression of NOX4 [8]. All these mitochondrial effects are gender dependent. Estrogens decrease endothelial damage as they participate in the regulation of vasodilation by activating endothelial NO synthase and increasing NO levels and reducing sympathetic activation and angiotensin II [9].

\section{Mineral Metabolism Contribution to CRMetS}

An approach to the CRMetS can also be made through the role of the nuclear receptors, with the participation of the vitamin D receptor (VDR) and its relationship with the parathyroid hormone (PTH). During chronic kidney damage, a decrease in the ability to convert vitamin $\mathrm{D}$ in calcitriol and in the excretion of phosphorus leads to hypocalcemia. Consequently, levels of PTH increase, so in an attempt to compensate the loss of calcium, the renal absorption of calcium and vitamin $\mathrm{D}$ will increase along with an activation of the bone resorption [10]. High levels of PTH have been related to a higher prevalence of metabolic syndrome in nondiabetic patients with CRS [11]. 
Moreover, the lack of activation of VDR leads to increased levels of nuclear factor kappa B, tumor necrosis factor alpha, and interferon gamma; recruitment of macrophages; and increased risk of atherosclerosis [10]. In addition, it produces cell calcification, fibrosis, and left ventricular hypertrophy [12].

Due to this intimate relation, the phosphorous levels are also important to the vessel's health. Two consequences have been described due to the accumulation of phosphorous: 1) an increase in the levels of core-binding factor alpha 1 and osteocalcin, responsible for the arterial stiffening, and 2) the accumulation of fibrosis growth factor 23 (FGF23) [1]. FGF23 plays a crucial role in the CRS since it may be responsible for the left ventricle hypertrophy, reduced function, and cardiac fibrosis. Secreted by osteocytes, FGF23 requires the presence of $\alpha$-Klotho to bind to its receptor. A reduction in the circulating levels of $\alpha$-Klotho produced by the kidney has been proved to precede rising levels of PTH and FGF23 during chronic kidney disease; therefore, it may be considered as a marker of cardiovascular risk [13].

Another main character among the nuclear receptors is the mineralocorticoid receptor activated by aldosterone, which promotes the reabsorption of sodium and upregulation of angiotensin II. Under these circumstances, transforming growth factor- $\beta 1$ increased the reinforcing of the loop, leading to fibrosis, increase in angiotensinogen, and apoptosis [11]. In this sense, the mineralocorticoid receptor antagonism may improve these parameters, but more research is needed [7].

\section{Role of Microbiota in CRMetS}

Patients with end-stage renal disease (ESRD) show a different microbiota from the healthy individuals, characterized by the presence of bacteria that contains enzymes responsible for the formation, among others, of uremic toxins, such as indole and p-cresol. Indoxyl sulfate (IS) and p-cresyl sulfate may not be eliminated by the kidney of ESRD patients. IS has been demonstrated to have a profibrotic activity as well as the potential to induce oxidative stress. Moreover, IS can induce the hypertrophy of the left ventricle and the downregulation of aforementioned $\alpha$-Klotho. p-Cresyl sulfate, along with IS, may induce the renal production of angiotensinogen and reactive oxygen species together with the upregulation of MCP-1 (monocyte chemotactic protein 1). Interestingly, NO can be produced by oral microbiota and the regulation of its composition is being studied to contribute to the regulation of the NO system production [14]. Therefore, it is crucial in maintaining the vascular tone and the integrity of the endothelium, like in this pathology. In addition, obese patients exhibit a characteristic microbiota, capable of producing pro-inflammatory molecules that cross the intestinal barrier and act on the different organs, which would be worsening and accelerating this problem.

\section{Management of the CRMetS}

The key points that should be taken into account when handling this pathology are 1) the maintenance of vascular functionality, blocking the RAAS, and improving the bioavailable NO;2) glycemic control; 3) body weight control; and 4) "healthy" gut microbiota. The treatment of these patients should accompany the use of RAAS blockers (angiotensin-converting enzyme inhibitors and angiotensin-receptor antagonist), together with metformin for glycemic control. Also, it would be advisable to use the sodium-glucose cotransporter-2 inhibitors that, in addition to helping glycemic control, have cardioprotective effects. Sodium-glucose cotransporter-2 inhibitors block the reabsorption of glucose, promoting glycosuria, natriuresis, and, consequently, a net loss of calories, body weight, and an improvement in blood pressure [15].

These pharmacotherapies should be accompanied by exercise and an adequate diet, to promote weight loss and a non-pro-inflammatory gut microbiota. The diet of these patients has to include salt and protein restriction, vegetable intake, and the use of pro-, pre-, and synbiotics [14].

\section{Conclusions and Future Perspectives}

The constellation of metabolic risk factors as the CRMetS considers obesity as a pivotal factor in its development. More research would be needed to enable us to understand and detect early CRMetS. In the meantime, the treatment of these patients should combine drug use, with exercise, and a proper diet to decline the adiposity and to reverse the pro-inflammatory cycle.

\section{Statement of Ethics}

The paper is exempt from Ethical Committee approval. No experiments were needed for the manuscript.

\section{Conflict of Interest Statement}

The authors have no conflicts of interest to declare. 


\section{Funding Sources}

Research conducted for this publication was supported by Ministerio de Economía y Competitividad de España (BFU201678951-R, BFU2017-90578-REDT), Comunidad de Madrid (Spain) (B2017/BMD-3684), to G.M.G.

\section{Author Contributions}

A.G.C., A.I.L., and G.M.G. drafted and revised the paper. All authors approved the final version of the manuscript.

\section{References}

1 Costanzo MR. The cardiorenal syndrome in heart failure. Heart Fail Clin. 2020;16(1):8197.

2 Sowers JR. Metabolic risk factors and renal disease. Kidney Int. 2007;71(8):719-20.

3 Pellegrinelli V, Carobbio S, Vidal-Puig A. Adipose tissue plasticity: how fat depots respond differently to pathophysiological cues. Diabetologia. 2016;59(6):1075-88.

4 Pazos F. Range of adiposity and cardiorenal syndrome. World J Diabetes. 2020;11(8):32250.

5 Chaudhary K, Malhotra K, Sowers J, Aroor A. Uric Acid - key ingredient in the recipe for cardiorenal metabolic syndrome. CardioRenal Med. 2013;3(3):208-20.

6 Gupta D, Brietzke S, Hayden MR, Kurukulasuriya LR, Sowers JR. Phosphate metabolism in cardiorenal metabolic disease. Cardiorenal Med. 2011;1(4):261-70.
7 Cabandugama PK, Gardner MJ, Sowers JR. The renin angiotensin aldosterone system in obesity and hypertension: roles in the cardiorenal metabolic syndrome. Med Clin North Am. 2017;101(1):129-37.

8 Aroor AR, Mandavia C, Ren J, Sowers JR, Pulakat L. Mitochondria and oxidative stress in the cardiorenal metabolic syndrome. Cardiorenal Med. 2012;2(2):87-109.

9 Jia G, Aroor AR, Sowers JR. Estrogen and mitochondria function in cardiorenal metabolic syndrome [Internet]. Progress in molecular biology and translational science. 1st ed. Elsevier Inc.; 2014. Vol. 127; p. 229-249.

10 Ronco C, Cozzolino M. Mineral metabolism abnormalities and vitamin $\mathrm{D}$ receptor activation in cardiorenal syndromes. Heart Fail Rev. 2012 Mar; 17(2):211-20.

11 Saab G, Whaley-Connell A, Bombeck A, Kurella Tamura M, Li S, Chen SC, et al. The association between parathyroid hormone levels and the cardiorenal metabolic syndrome in non-diabetic chronic kidney disease. Cardiorenal Med. 2011;1(2):123-30.
12 Chun Li Y. Vitamin d receptor signaling in renal and cardiovascular protection. Semin Nephrol. 2013 Sep;33(5):433-47.

13 Law JP, Price AM, Pickup L, Radhakrishnan A, Weston C, Jones AM, et al. Clinical potential of targeting fibroblast growth factor- 23 and aKlotho in the treatment of uremic cardiomyopathy. J Am Heart Assoc. 2020 Apr 7; 9(7):e016041.

14 Cosola C, Rocchetti MT, Cupisti A, Gesualdo L. Microbiota metabolites: pivotal players of cardiovascular damage in chronic kidney disease. Pharmacol Res 2018;130:132-42.

15 Jindal A, Brietzke S, Sowers JR. Obesity and the cardiorenal metabolic syndrome: therapeutic modalities and their efficacy in improving cardiovascular and renal risk factors. Cardiorenal Med. 2012 Nov 26;2(4):314-27. 